\title{
Approaches to Self-Powered Biochemical Sensors for In- Vivo Applications
}

\author{
E.M. Yeatman \\ Department of Electrical \& Electronic Engineering \\ Imperial College London \\ London SW7 2AZ \\ +44-20-7594-6204 \\ e.yeatman@imperial.ac.uk
}

\author{
D. O'Hare, C. Dobson, E. Bitziou \\ Department of Bioengineering \\ Imperial College London \\ London SW7 2AZ \\ +44-20-7594-5173 \\ d.ohare@imperial.ac.uk
}

\begin{abstract}
The requirement for electrical power is a major limitation in the development of biosensors for in-body applications. This paper considers motion powered energy harvesting devices for in-body use, showing that power levels are unlikely to exceed a few microwatts for devices of acceptable size. As a low power sensor, we are developing $\mathrm{pH}$ detectors based on metal-metal oxide electrodes, such as iridium oxide. The power requirement of these devices is considered, including signal conditioning, storage and transmission. A hybrid powering scheme of energy harvesting and wireless power delivery for data transmission is proposed.
\end{abstract}

\section{Keywords}

Energy scavenging, biosensors, MEMS

\section{INTRODUCTION}

Compact, long lasting power sources are a key enabler for wireless devices in general, and particularly so for wireless sensor nodes. Sensor nodes will often have very limited size, and for sensor networks in which a large number of nodes are desirable, battery replacement or recharging can become an unacceptable system maintenance burden. Therefore energy scavenging power sources are being extensively investigated for such applications [1]. The main sources of ambient energy are sunlight or other ambient light, temperature differences, and motion. In this paper we will concern ourselves primarily with the latter.

For biomedical applications, body mounted and implanted sensors present unique powering challenges. For the latter in particular, battery replacement is highly undesirable, and recharging via induction loops is likely to be slow and inconvenient. Furthermore, the efficiency of contactless power transfer by induction drops rapidly as the receiving coil is reduced in size. Biocompatibility of battery materials is also a concern for implanted devices. For these reasons energy scavenging is highly attractive for implanted biosensors.

\section{BODY MOTION ENERGY HARVESTING}

The power levels available from motion have been extensively analysed [2]. The ultimate limits depend on the size of the scavenging device, and on the frequency and amplitude of the motion concerned. In general the motion scavengers are so-called inertial devices, i.e. having an internal proof mass $m$, moving over an internal range $\pm Z_{\mathrm{L}}$, within a frame attached to a moving source.

Permission to make digital or hard copies of all or part of this work for personal or classroom use is granted without fee provided that copies are not made or distributed for profit or commercial advantage and that copies bear this notice and the full citation on the first page. To copy otherwise, to republish, to post on servers or to redistribute to lists, requires prior specific permission and/or a fee.

BodyNets 2008, March 13-15 Tempe, Arizona, USA

Copyright (C) 2008 ICST 978-963-9799-17-2

DOI 10.4108/ICST BODYNETS2008.2973
If the source motion has amplitude $\mathrm{Y}_{\mathrm{o}}$ and frequency $f$, then the maximum power is [2]:

$$
\mathrm{P}_{\text {max }}=4 \pi^{2} \mathrm{mf}^{3} \mathrm{Y}_{\mathrm{o}} \mathrm{Z}_{\mathrm{l}}
$$

For a device of dimensions $\mathrm{s} \times \mathrm{s} \times 2 \mathrm{~s}$, having a cubic proof mass of volume $s^{3}$ and density $\rho$, and internal motion amplitude $Z_{L}=$ $\pm \mathrm{s} / 2$, we can derive

$$
\mathrm{P}_{\max }=2 \pi^{2} \rho s^{4} \mathrm{f}^{3} \mathrm{Y}_{\mathrm{o}}
$$

To determine how much power can be obtained for a given device size, we must consider the nature of the available motion. We choose three examples:

(i) vertical motion of torso during walking: $\mathrm{f}=1 \mathrm{~Hz}, \mathrm{Y}_{\mathrm{o}}=4 \mathrm{~cm}$

(ii) chest motion from normal breathing: $\mathrm{f}=0.3 \mathrm{~Hz}, \mathrm{Y}_{\mathrm{o}}=1 \mathrm{~cm}$

(iii) heart motion: $\mathrm{f}=1 \mathrm{~Hz}, \mathrm{Y}_{\mathrm{o}}=0.5 \mathrm{~cm}$

From these values and using (2) we can obtain the achievable power levels as a function of device dimensions. These are plotted in Fig 1, for a proof mass density $\rho=10 \mathrm{~g} / \mathrm{cc}$.

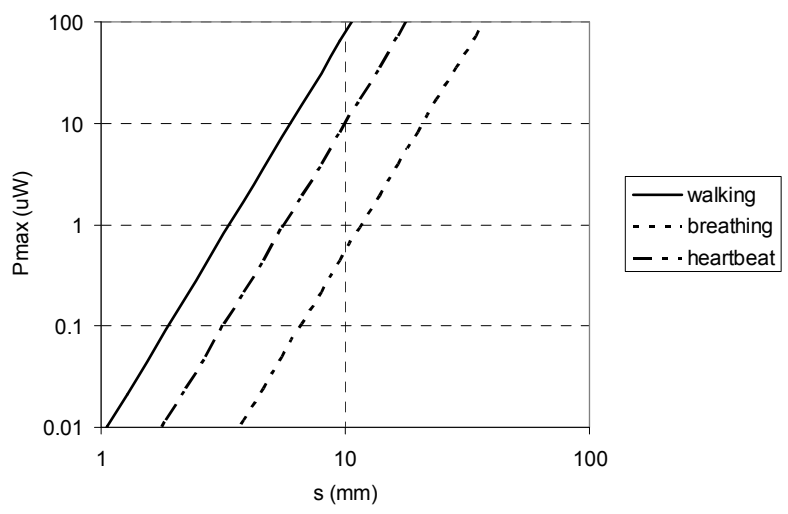

Figure 1. Maximum power levels vs. device dimension $s$, for different body motion sources as indicated.

It can be seen that for typical levels of body motion, continuous power levels from a harvester below $1 \mathrm{~cm}^{3}$ are likely to be at most about a few microwatts. Reported energy harvesters to date reach levels typically below $10 \%$ of these theoretical maxima.

\section{POWER REQUIREMENTS}

The power levels of Fig. 1 must be compared to the sensor requirements. The power demand relates to: powering of the 
sensor itself; signal conditioning, including digitization and possibly storage; and communication.

We have considered the specific case of electrochemical sensors. These show great potential for biomedical monitoring [3], since they have modest instrumental requirements and can be easily miniaturized. They fall into two broad classes: potentiometric passive devices, where potential is related logarithmically to concentration, and amperometric devices, where an external applied potential leads to a current linearly related to concentration. The former is inherently better suited to ultra-low power applications. Here we consider potentiometric $\mathrm{pH}$ sensors.

Measurement of $\mathrm{pH}$ is important in the study of tissue metabolism and is an indicator of tissue nutrition. Applications are therefore broad in both acute medicine (anaesthesia, plastic surgery) and chronic applications (wound healing, diabetes). The expected range is $4<\mathrm{pH}<8$ in most applications, though somewhat narrower in blood. Whilst glass membrane $\mathrm{pH}$ sensors are established technology, they are mechanically fragile, have slow response times and are not amenable to the extreme miniaturisation required for implantation. An attractive alternative is to use $\mathrm{pH}$ sensitive metal-metal oxide film sensors [4], particularly hydrated iridium oxide, since they are mechanically stable and compatible with CMOS processing, an important consideration for long-term development.
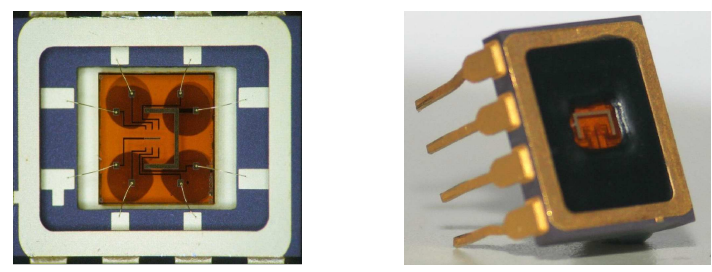

Figure 2. Microsensor array platform: chip prior to packaging (I); completed device in DIL package (r).

Figure 2 shows the sensors we are developing. These show intrinsic selectivity and are operated entirely passively. All that is required is a high input impedance circuit to read the voltage signal. This ensures that no significant current is drawn from the sensor, in order to prevent Faradaic reactions either within the film or at the surface. Intrinsic sensitivity is typically $75-80 \mathrm{mV}$ per pH unit. Fig. 3 shows examples of calibration working curves obtained from these devices. From these results we can deduce some circuit requirements: an operating range of 0 to $0.5 \mathrm{~V}$ is sufficient for the $\mathrm{pH}$ range of interest, and 8 bit digitization will provide $\mathrm{pH}$ resolution of better than 0.02 across the entire range.

The next requirement is for signal conditioning. Since the output is in a suitable voltage range for direct digitization, we can in principle dispense with an interface analogue amplifier, and connect the sensor directly to an analog-to-digital converter (ADC) of suitable range. Fortunately, very low power ADCs have been reported; in [5], power dissipation below $1 \mu \mathrm{W}$ was achieved for 8 bit sampling at $4 \mathrm{kS} / \mathrm{s}$, although for an input voltage swing of only $0.1 \mathrm{~V}$. For the $\mathrm{pH}$ sensor application, a few samples/sec are likely to be sufficient, so sub- $\mu \mathrm{W}$ ADC power should remain achievable. Temporary data storage into, for example, flash memory, is likely to require milliwatts during writing. However, microwatt standby power levels are typical, and sub-microsecond write cycles would allow average write power to be also in the microwatt range.

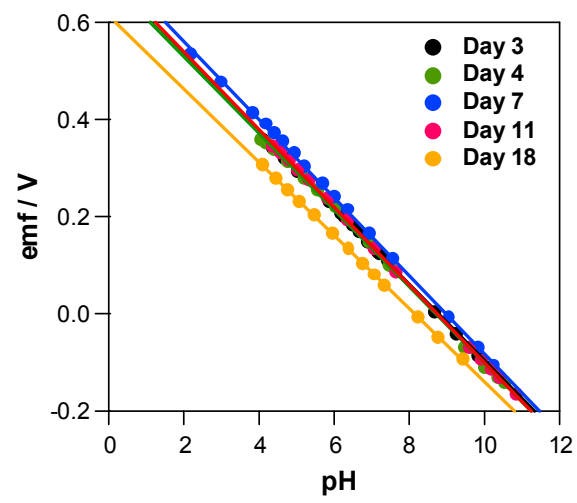

Figure 3. Sensor calibration working curves obtained in buffer over 18 days.

Data transmission from within the body, even at minimal bit rate, requires power levels well above $1 \mu \mathrm{W}$ with present technology. However, a hybrid scheme can be proposed in which sets of sample values are stored locally, for periodic transmission. This communication link could be supported by power delivered wirelessly by the reading unit, as is done with passive RFID [6]

In conclusion, self-powered electrochemical biosensors for implanted applications would appear to be feasible, using motion energy harvesters of a few mm dimension, with ultra low power digitization and storage, and data transmission based on delivered power.

\section{ACKNOWLEDGMENTS}

This work was supported by the Engineering \& Physical Sciences Research Council under the Biosensornet project.

\section{REFERENCES}

[1] J. A. Paradiso and T. Starner, "Energy scavenging for mobile and wireless electronics," Pervasive Computing, IEEE, vol. 4, pp. 18-27, 2005.

[2] P. D. Mitcheson, T. C. Green, E. M. Yeatman, and A. S. Holmes, "Architectures for vibration-driven micropower generators," Microelectromechanical Systems, Journal of, vol. 13, pp. 429-440, 2004.

[3] B. A. Patel, C. A. Anastassiou, and D. O'Hare, "Biosensor design and interfacing," in Body sensor networks, G.-Z. Yang, Ed. New York: Springer-Verlag, 2006.

[4] D. O'Hare, K. H. Parker, and C. P. Winlove, "Metal-metal oxide $\mathrm{pH}$ sensors for physiological application," Med. Eng. \& Physics, vol. 28, pp. 982-988, 2006.

[5] J. Sauerbrey, D. Schmitt-Landsiedel, and R. Thewes, "A 0.5V 1-/spl mu/W successive approximation ADC," Solid-State Circuits, IEEE Journal of, vol. 38, pp. 1261-1265, 2003.

[6] B. Jiang, J. R. Smith, M. Philipose, S. Roy, K. Sundara-Rajan, and A. V. Mamishev, "Energy Scavenging for Inductively Coupled Passive RFID Systems," Instrumentation and Measurement, IEEE Trans. on, vol. 56, pp. 118-125, 2007. 